\title{
A!
}

This is an electronic reprint of the original article.

This reprint may differ from the original in pagination and typographic detail.

Karakoç, Alp; Paltakari, Jouni; Taciroglu, Ertugrul

\section{Data-Driven Computational Homogenization Method Based on Euclidean Bipartite Matching}

Published in:

Journal of Engineering Mechanics

DOI:

10.1061/(ASCE)EM.1943-7889.0001708

Published: 01/02/2020

Document Version

Peer reviewed version

Please cite the original version:

Karakoç, A., Paltakari, J., \& Taciroglu, E. (2020). Data-Driven Computational Homogenization Method Based on Euclidean Bipartite Matching. Journal of Engineering Mechanics, 146(2), [04019132].

https://doi.org/10.1061/(ASCE)EM.1943-7889.0001708

This material is protected by copyright and other intellectual property rights, and duplication or sale of all or part of any of the repository collections is not permitted, except that material may be duplicated by you for your research use or educational purposes in electronic or print form. You must obtain permission for any other use. Electronic or print copies may not be offered, whether for sale or otherwise to anyone who is not an authorised user. 
A DATA-DRIVEN COMPUTATIONAL HOMOGENIZATION METHOD BASED ON EUCLIDEAN BIPARTITE MATCHING

\section{Alp Karakoç ${ }^{1}$, Jouni Paltakari ${ }^{2}$, Ertugrul Taciroglu ${ }^{3}$}

${ }^{1}$ Postdoctoral researcher, Civil \& Env. Engineering Department, University of California Los Angeles, 90095 CA, USA. Email: akarakoc@alumni.ucla.edu

${ }^{2}$ Professor, Aalto University, Department of Bioproducts and Biosystems, FI-00076 AALTO,

$8 \quad$ FINLAND. Email: jouni.paltakari@aalto.fi

$9{ }^{2}$ Civil \& Env. Engineering Department, University of California Los Angeles, 90095 CA, USA.

10 Email: etacir@ucla.edu

\section{ABSTRACT}

Image processing methods combined with scanning techniques-e.g., microscopy or microtomography_are now being frequently used for constructing realistic microstructure models that can be used as representative volume elements (RVEs) to better characterize heterogeneous material behavior. As a complement to those efforts, the present study introduces a computational homogenization method that bridges the RVE and material-scale properties in situ. To define the boundary conditions properly, an assignment problem is solved using Euclidean bipartite matching through which the boundary nodes of the RVE are matched with the control nodes of the rectangular prism bounding the RVE. The objective is to minimize the distances between the control and boundary nodes, which when achieved enables the bridging of scale-based features of both virtually generated and image-reconstructed domains. Following the minimization process,

24 periodic boundary conditions can be enforced at the control nodes, and the resulting boundary 25 value problem can be solved to determine the local constitutive material behavior. To verify the proposed method, virtually generated domains of closed-cell porous, spherical particle and fiber reinforced composite materials are analyzed, and the results are compared with analytical HashinShtrikman and Halpin-Tsai methods. The percent errors are within the ranges from $0.04 \%$ to $3.3 \%$, from $2.7 \%$ to $14.9 \%$, and from $0.5 \%$ to $13.2 \%$ for porous, particle and fiber reinforced composite

30 materials, respectively, indicating that the method has a promising potential in the fields of image-

31 based material characterization and computational homogenization. 
KEYWORDS: Microscopy, micro-tomography, representative volume element, assignment

34 problem, material characterization, computational homogenization.

\section{INTRODUCTION}

Image processing methods combined with scanning techniques-e.g., laser scanning confocal microscopy, micro-computed tomography $(\mu \mathrm{CT})$, scanning electron microscopy (SEM), magnetic resonance imaging (MRI) to name a few-are gaining attention due to their capabilities in determining surface and volumetric properties, chemical compositions, and mechanical and

42 directional features of materials (Nazar et al. 1996; Huang and Wei 2010; Duval et al. 2014).

43 Although there are various complexities in image acquisition, segmentation and rendering and

44 needs for considerable user interaction, these methods have been paving the way to highly refined

45 levels of data-driven material characterization (Hollister and Kikuchi 1994; Terada et al. 1997). In

46 addition to the innovations and developments in the image acquisition systems, numerous

47 segmentation and rendering techniques have been also developed to process the obtained image

48 data and extract high-fidelity models (Takano et al. 2003; Legrain et al. 2011; Lopez et al. 2014;

49 Ren et al. 2015). In most of these techniques, the solution domain is discretized with the aim of

50 minimum data loss to reconstruct so-called realistic representative volume element (RVE), to

51 which the mechanical and physical properties, and boundary conditions are assigned (Lian et al.

52 2013; Bargmann et al. 2018). Boundary volume problem (BVP) can be then solved-e.g., using

53 the finite element method in the computational homogenization framework - over the RVE

54 boundaries to bridge the micro- and material-scale properties for the effective mechanical

55 properties (Geers et al. 2010; Karakoc et al. 2017). However, due to unstructured nature of the 56 reconstructed RVEs directly from the images, defining the RVE boundaries and node mapping for 57 computational homogenization - especially, in the case of periodic boundary definitions - can 58 turn into a nontrivial process (Lian et al. 2013; Nguyen and Noels 2014). To the authors' 59 knowledge, various boundary condition enforcement methods are available in the literature 60 including the local implementation method (Tyrus et al. 2007), master/slave approach (Yuan and 61 Fish 2008), weak periodicity (Larsson et al. 2011). Most of these methods have been successfully 62 tested on RVEs composed of inclusions embedded in matrix; however, there still needs to be 
63 development in enforcement methods - e.g., in case of the dominant presence of pores on the RVE

64 boundaries (see, Figure 1) (Nguyen et al. 2012).

In consideration to the challenges in data-driven material characterization, the present study

67 introduces a computational homogenization method, through which periodic boundary conditions are enforced via total distance minimization of control and boundary node sets as shown in Figure 2. This, in turn, enables computational homogenization of domains represented by arbitrary meshes - e.g., in-situ reconstructed domains via image processing methods, and bridging of scalebased features of both image-reconstructed and virtually generated domains. The present study is

72 therefore expected to advance the current state of the art towards accurate material characterization 73 with low computational costs.

\section{METHODOLOGY}

As illustrated in Figures 2 and 3, the BVP is defined at the RVE scale first wherein the boundary nodes $(p)$ on the RVE boundaries $(\partial \omega)$ are matched with the control nodes $(q)$, which are uniformly discretized and represented in the form of corner, edge, and surface nodes on the boundaries $(\partial \Gamma)$ of its bounding rectangular prism. The grid spacing on $\partial \Gamma$, is taken as the mean value $d_{\text {mean }}$ of the closest-pair distances on the corresponding $\partial \omega$ surface. Simply, the closest-pair

82 distance is calculated as the Euclidean distance between the reference $p$ node and its nearest neighbor. After this step, alpha shapes method is used to reduce the $q$ set size. Thereafter, one-toone matching between $p$ and $q$ sets are carried out through a distance minimization technique

85 known as the Euclidean bipartite matching. Eventually, periodic boundary conditions are enforced, rigid body rotations are eliminated, and the BVP is solved in the computational homogenization

87 scheme based on the first-order strain-driven homogenization method.

\section{Control node reduction}

91 In order to reduce the excessive amount of control nodes-i.e., $q$ node set size reduction, especially

92 for the RVE boundaries with pores, the alpha shapes method is used (Edelsbrunner et al. 1983;

93 Edelsbrunner 1995). As illustrated in Figure 4, the boundary nodes $p$ obtained from the geometry 
94 file is used to construct (I) control node sets as grid points by means of $d_{\text {mean }}$ and (II) Delaunay 95 triangulation where the elements (polygons in 2D and polyhedrons in 3D), the circumradii $R$ of 96 which exceeds the alpha shape value (taken to be $d_{\text {mean }}$ in the present study), are discarded

97 (Cerquaglia et al. 2017). Thereafter, the control nodes are refined by eliminating the control nodes 98 in the region of excessive elements identified with the alpha shapes method.

\section{Euclidean bipartite matching of the boundary and control nodes}

The core of the proposed method is based on Euclidean bipartite matching of the boundary nodes denoted with $p$ and with $q$, as depicted in Figure 2. The total distance between the node sets are minimized to determine an optimal matching and thus, to obtain one-to-one correspondence between $p$ and $q$. As the first step, a cost matrix based on the Euclidean distance of each $(p, q)$ combination is generated, which results to an $N \times N$ matrix where $N$ is equal to the set length of $p$-i.e.,

$$
\left[\begin{array}{cccc}
\mathbf{d}\left(p_{1}, q_{1}\right) & \mathbf{d}\left(p_{1}, q_{2}\right) & \ldots & \mathbf{d}\left(p_{1}, q_{n}\right) \\
\mathbf{d}\left(p_{2}, q_{1}\right) & \ldots & \ldots & \ldots \\
\ldots & \ldots & \ldots & \ldots \\
\mathbf{d}\left(p_{n}, q_{1}\right) & \ldots & \ldots & \mathbf{d}\left(p_{n}, q_{n}\right)
\end{array}\right]
$$

Then, optimal permutation of matched nodes is discerned based on their total Euclidean distances through the minimization problem

$$
T=\min \sum_{\Pi} \mathbf{d}(p, q)
$$
where $\mathbf{d}$ is the Euclidean distance function for two nodes, $T$ is the total Euclidean distance and $\Pi$ is the permutations that abide a one-to-one correspondence. Under these circumstances, there should be only one matching pair for each $p$ and $q$, otherwise the one-to-one correspondence condition is violated. The problem in Eq. (2) can be solved with-e.g., Monte-Carlo simulations generating random possible solutions and selecting the solution with the minimum cost, various heuristics for determining the optimum cost, or schemes relaxing the given problem into a series of problems for each of which an optimal solution is obtained (Rendl 1988; Hung and Rom 1980; Karakoc and Taciroglu 2017). In the present study, Monte Carlo simulations are used, for which the solution provides one-to-one matching of control and boundary nodes that exhibit the shortest 
121 total distance $T$, as shown in Figure 2. Following the matching process, there are two possible

122 options in defining the boundary conditions: $(i)$ to use directly the boundary nodes $p$ obtained as a 123 result of the minimization, or (ii) further, to tie the degrees of freedom of the one-to-one matched

124 nodes and to use the control nodes $q$ (-i.e., $\vec{u}_{p}=\vec{u}_{q}$ ). In the present study, the latter option is 125 preferred.

\section{First-order computational homogenization}

In the present study, a first-order strain driven homogenization is utilized to determine the elastic properties. As shown in Figure 5, the macro-strain $\mathbf{e}^{\mathrm{M}}$ is known a priori where the associated macro-stress $\mathbf{s}^{\mathrm{M}}$ is computed through volume averaging of the stress field at the RVE scale

132 (Hernández et al. 2014).

134 Here, the macro-strain $e_{i j}^{\mathrm{M}}$ for $i, j \in\{X, Y, Z\}$ is the given parameter and is used as the driving 135 parameter of the microscopic displacement field for the RVE so that

$$
\vec{u}^{\mathrm{m}}=\vec{r} \cdot \mathbf{e}^{\mathrm{M}}+\underline{\vec{u}} .
$$

137 The first addend of Eq. (3) on the right-hand side represents the macroscopic displacement

138 contribution, and the second represents the displacement fluctuation field $\underline{\vec{u}}$ due to heterogeneities

139 within the RVE (Geers et al. 2010). Here, $\vec{r}$ represents the position vector between two nodes and 140 the overall body is assumed to be composed of repeating rectangular prism bounding the RVEs. 141 Continuity conditions for the displacement field are satisfied at each adjacent boundary by taking 142 the relative positions of the control node sets $q$, which eliminates $\underline{\vec{u}}$.

144 In computational homogenization studies, the use of RVEs with periodic boundary conditions is a 145 common practice, for which the corresponding corner, edge and surface nodes are matched as 146 previously depicted in Figure 3, and suffices to represent the effective material deformation 147 (Karakoc 2018). Following this common practice, periodic boundary conditions are applied onto 148 the control nodes $q$ of the rectangular prism bounding the RVE. Here, it is important that the so149 called "periodic offset" caused by the distance between matched nodes is inevitable. 
152 In computational homogenization, Hill-Mandel principle gives the relationship between the micro153 and material scales such that

$$
\mathbf{s}^{\mathrm{M}}: \mathbf{e}^{\mathrm{M}}=\frac{1}{\Gamma} \int_{\Gamma} \mathbf{s}^{\mathrm{m}}: \mathbf{e}^{\mathrm{m}} \mathrm{d} \Gamma,
$$

155 for which superscripts $\mathrm{m}$ and $\mathrm{M}$ stand for micro- and material scales. The symbol (:) denotes the 156 inner product $\mathbf{a}: \mathbf{b}=a_{i j} b_{i j}$ for second-order tensors. By using the Gauss theorem, Eq. (4) can be 157 rewritten over $\partial \Gamma$ as

$$
\mathbf{s}^{\mathrm{M}}: \mathbf{e}^{\mathrm{M}}=\frac{1}{\Gamma} \int_{\partial \Gamma} \vec{t}^{\mathrm{m}} \cdot \vec{u}^{\mathrm{m}} \mathrm{d} \partial \Gamma,
$$

where $\vec{t}^{\mathrm{m}}$ is the micro-scale traction vector at $\partial \Gamma$. By plugging the boundary periodicity into Eq.

160 (5), we get

$$
\mathbf{s}^{\mathrm{M}}: \mathbf{e}^{\mathrm{M}}=\frac{1}{\Gamma} \int_{\partial \Gamma} \vec{t}^{\mathrm{m}} \cdot\left(\vec{r} \cdot \mathbf{e}^{\mathrm{M}}\right) \mathrm{d} \partial \Gamma+\frac{1}{\Gamma} \int_{\partial \Gamma} \vec{t}^{\mathrm{m}} \cdot \underline{\vec{u}} \mathrm{~d} \partial \Gamma,
$$

which can be rearranged into

$$
\mathbf{s}^{\mathrm{M}}: \mathbf{e}^{\mathrm{M}}=\frac{1}{\Gamma} \int_{\partial \Gamma}\left(\vec{t}^{\mathrm{m}} \otimes \vec{r}\right) \mathrm{d} \partial \Gamma: \mathbf{e}^{\mathrm{M}}+\frac{1}{\Gamma} \int_{\partial \Gamma} \vec{t}^{\mathrm{m}} \cdot \underline{\vec{u}} \mathrm{~d} \partial \Gamma .
$$

Here, the symbol $\otimes$ denotes the dyadic operator. The second integrand at the right-hand side vanishes in case of periodic boundary conditions. Hence, macro-scale stress $\mathbf{s}^{\mathrm{M}}$ can be expressed as the volume average of the micro-scale stress $\mathbf{s}^{\mathrm{m}}$ such that

$$
\mathbf{s}^{\mathrm{M}}=\frac{1}{\Gamma} \int_{\partial \Gamma}\left(\vec{t}^{\mathrm{m}} \otimes \vec{r}\right) \mathrm{d} \partial \Gamma=\frac{1}{\Gamma} \int_{\Gamma} \mathbf{s}^{\mathrm{m}} \mathrm{d} \Gamma,
$$

where $\Gamma$ is the total volume of the rectangular prism bounding the RVE. Then, the given strains $\mathbf{e}^{\mathrm{M}}$ and the computed stresses $\mathbf{s}^{\mathrm{M}}$ at the material scale can be then combined. Eventually, by means of a least-squares minimization of all six distinct deformation modes in three-dimensional space

171 (three axial and three shear loading modes), the compliance $\mathbf{C}^{\mathrm{M}}$ is obtained as

$$
\pi\left(C_{11}, . ., C_{66}\right)=\sum_{i=1}^{n}\left\|\mathbf{e}_{i}^{\mathrm{M}}-\mathbf{C}^{\mathrm{M}}: \mathbf{s}_{i}^{\mathrm{M}}\right\|^{2}
$$

173 where $i$ refers to the number of experiments (Karakoç et al. 2013; Sjolund et al. 2014). 
In order to understand the capability of the algorithm, effect of RVE mesh size and control node number on the convergence of effective elastic properties and central processing unit (CPU) time

178 study are investigated. For this purpose, the closed cell porous RVEs, which can be sometimes computationally expensive due to the non-proportional number of pores in the opposing surfaces, have been generated. The matrix material is selected to be epoxy resin with $E_{\mathrm{m}}=3000 \mathrm{MPa}$ and $v_{\mathrm{m}}=0.38$. Two sets of simulations have been carried out: (I) one set of simulations with different number of seeds per edge $s=\{2,2.2,2.5,2.9,3.3,4,5,6.7,10,20,40\}$ and corresponding mesh sizes $e=\{46768,49178,50782,53388,57556,68388,69961,86193,102843,185795,558311\}$,

184 three RVEs of which are depicted in Figure 6a, and (II) one set of simulations with different control node numbers $q=\{152,568,1224,2402,2906,3458,3628\}$, three RVEs of which are shown in Figure 7a.

As seen in Figure 6b, there is a positive correlation between the mesh size and CPU time. Despite this trade-off, the effective elastic properties do not vary with increasing mesh size-e.g., after RVE with 185795 elements referring to edge seeding number of 20. Even though the element size in the finest RVE mesh is almost triple reaching 558311 elements, the maximum percentile difference is low and obtained as $3.8 \%$ for $E_{33}$. However, there is extreme increase in the CPU time from 423 seconds to 1992 seconds for these two cases. Based on the results, element size is

194 based on the edge seeding number of 20 in the present study. Thereafter, the effect of control node number on the effective elastic properties and CPU time have been investigated. The RVE is selected to be the one with 185795 elements-i.e, edge seeding number of 20. As seen in Figure $7 \mathrm{~b}$, there is a fast convergence for both the effective elastic properties and CPU time with a maximum value of 423 seconds showing that the present framework works efficiently with even moderate numbers of control nodes.

\section{METHOD VERIFICATION}

Previously conducted analytical, numerical and experimental studies and their results on closed cell porous materials, particle and fiber reinforced composites, the microstructures of which are 
illustrated in Figure 1, are used so as to verify the present method (Hashin and Shtrikman 1963; Halpin 1969; Kushnevsky et al. 1998; Segurado and Llorca 2002; Babu et al. 2018).

\section{Case study: Closed cell porous and spherical particle reinforced composites}

In order to verify the present method for closed cell porous and spherical particle reinforced composites, RVE geometries are formed based on the procedure provided by Segurado and Llorca

212 (Segurado and Llorca 2002). In line with this procedure, two cases are considered, for which unit 213 cubes $(1 \times 1 \times 1)$ containing 30 non-overlapping randomly distributed reinforcing particles and 214 voids are generated with an in-house random sequential adsorption (RSA) code (Evans 1993). In 215 this code, if the particles or voids do not overlap with the previous ones, they remain fixed till the 216 end of the computational process reaching the desired particle or void volume fractions $217 V_{\mathrm{f}}=\left(\sum_{i=1}^{30} 4 / 3 \pi r_{i}^{3}\right)$ of the RVEs. In this case study, $V_{\mathrm{f}}$ is taken to be within the range from $10 \%$ to $21828 \%$. The generated RVE domains are then discretized by using C3D4 general-purpose tetrahedral 219 elements of ABAQUS (Hibbitt et al. 1992). The matrix material is selected to be epoxy resin with $220 E_{\mathrm{m}}=3000 \mathrm{MPa}$ and $v_{\mathrm{m}}=0.38$ and the reinforcing particles are chosen to be glass with $E_{\mathrm{f}}=70000$ $221 \mathrm{MPa}$ and $v_{\mathrm{f}}=0.20$ in case of particle reinforced composites. In case of voids, $E_{\mathrm{f}}$ is taken to be 222 arbitrarily small (-i.e., $\sim 0 \mathrm{~Pa}$ ). Due to heterogeneities in the RVEs, three numerical simulations 223 are conducted with the ABAQUS Standard finite element solver for each case and the simulation 224 results are compared with the analytical Hashin-Shtrikman bounds for both the closed cell porous 225 materials and the particle reinforced composites. Under the assumption of linear elastic isotropic 226 phases, these bounds for bulk $K$ and shear $G$ moduli are given as (Hashin and Shtrikman 1963; 227 Kushnevsky et al. 1998)

$$
\begin{gathered}
K_{\mathrm{c}}^{\mathrm{u}}=K_{\mathrm{f}}+\frac{\left(K_{\mathrm{m}}-K_{\mathrm{f}}\right)\left(3 K_{\mathrm{f}}+4 G_{\mathrm{f}}\right)\left(1-V_{\mathrm{f}}\right)}{\left(3 K_{\mathrm{f}}+4 G_{\mathrm{f}}\right)+3\left(K_{\mathrm{m}}-K_{\mathrm{f}}\right) V_{\mathrm{f}}}, \\
K_{\mathrm{c}}^{1}=K_{\mathrm{m}}+\frac{\left(K_{\mathrm{f}}-K_{\mathrm{m}}\right)\left(3 K_{\mathrm{m}}+4 G_{\mathrm{m}}\right) V_{\mathrm{f}}}{\left(3 K_{\mathrm{m}}+4 G_{\mathrm{m}}\right)+3\left(K_{\mathrm{f}}-K_{\mathrm{m}}\right)\left(1-V_{\mathrm{f}}\right)}, \\
G_{\mathrm{c}}^{\mathrm{u}}=G_{\mathrm{f}}+\frac{5 G_{\mathrm{f}}\left(G_{\mathrm{m}}-G_{\mathrm{f}}\right)\left(3 K_{\mathrm{f}}+4 G_{\mathrm{f}}\right)\left(1-V_{\mathrm{f}}\right)}{5 G_{\mathrm{f}}\left(3 K_{\mathrm{f}}+4 G_{\mathrm{f}}\right)+6\left(G_{\mathrm{m}}-G_{\mathrm{f}}\right)\left(K_{\mathrm{f}}+2 G_{\mathrm{f}}\right) V_{\mathrm{f}}},
\end{gathered}
$$




$$
G_{\mathrm{c}}^{1}=G_{\mathrm{m}}+\frac{5 G_{\mathrm{m}}\left(G_{\mathrm{p}}-G_{\mathrm{m}}\right)\left(3 K_{\mathrm{m}}+4 G_{\mathrm{m}}\right) V_{\mathrm{f}}}{5 G_{\mathrm{m}}\left(3 K_{\mathrm{m}}+4 G_{\mathrm{m}}\right)+6\left(G_{\mathrm{f}}-G_{\mathrm{m}}\right)\left(K_{\mathrm{m}}+2 G_{\mathrm{m}}\right)\left(1-V_{\mathrm{f}}\right)} .
$$

232 Here, the superscripts $u$ and $l$ denote the upper and lower bounds whereas subscripts $c, f$, and $m$

233 refer to composite, filling (particle or void), and matrix materials, respectively. The effective

234 Young's modulus, Poisson's ratio and shear modulus values of the composite are then expressed 235 as $E_{\mathrm{c} 11}=E_{\mathrm{c} 22}=E_{\mathrm{c} 33}=9 K_{\mathrm{c}} G_{\mathrm{c}} /\left(3 K_{\mathrm{c}}+G_{\mathrm{c}}\right), \quad v_{\mathrm{c} 12}=v_{\mathrm{c} 13}=v_{\mathrm{c} 23}=\left(3 K_{\mathrm{c}}-2 G_{\mathrm{c}}\right) /\left(2\left(3 K_{\mathrm{c}}+G_{\mathrm{c}}\right)\right), \quad G_{\mathrm{c} 12}=G_{\mathrm{c} 13}=G_{\mathrm{c} 23}=G_{\mathrm{c}}$, 236 respectively.

\section{Effective elastic properties of closed cell porous materials}

Void presence in materials is generally undesired and can drastically degrade the stiffness and strength properties. Therefore, to understand the effect of voids on the effective elastic properties of closed cell porous materials, all six distinct loading modes are simulated as seen in Figure 8.

243 The least-squares minimization problem of Eq. (9) is then solved to obtain the compliance,--hence

$244 E_{\mathrm{c} 11}, E_{\mathrm{c} 22}, E_{\mathrm{c} 33}, G_{\mathrm{c} 12}, G_{\mathrm{c} 13}, G_{\mathrm{c} 23}$, and $v_{\mathrm{c} 12}, v_{\mathrm{c} 13}, v_{\mathrm{c} 23}$.

Both the proposed method and the analytical solution show that there is a negative influence of voids on the elastic properties with a decrease of $28-29 \%$ for Young's moduli, $27 \%$ for shear moduli and 5\% for Poisson's ratios within the investigated volume fraction range, as listed in Table 1. The percent errors for Young's moduli, shear moduli, and Poisson's ratios between the proposed and analytical solutions are $0.04 \%-2.9 \%, 0.1 \%-2.5 \%, 0.1 \%-3.3 \%$, respectively.

\section{Effective elastic properties of spherical particle reinforced composites}

254 For the particle reinforced composite simulations, particles and matrix are assumed to be strongly bonded and only the effects of particle volume fraction on the effective elastic properties are examined. In order to deviate from the conventional computational homogenization methods, the

257 domain is discretized so that arbitrary meshes are generated on the RVE boundaries $\partial \omega$, as 258 illustrated in Figure 9. Both uniaxial and shear deformation modes are simulated to obtain the 259 effective elastic parameters listed in Table 2. 
261 The results show the reinforcing effect of particles on the elastic properties with an increase of 67-

$26269 \%$ for Young's moduli, 65-66\% for shear moduli, and a decrease of 8-13\% for Poisson's ratios

263 within the investigated volume fraction range. The decrease in $v$ is due to $v_{\mathrm{f}}<v_{\mathrm{m}}$. The percent

264 errors for Young's moduli, shear moduli, and Poisson's ratios between the proposed and analytical 265 solutions are 3.0\%-17.6\%, 2.7\%-15.3\%, 11.2\%-14.9\%, respectively.

\section{Case study: Fiber reinforced composites}

For the verification purpose of the model for the fiber reinforced composites, Halpin-Tsai equations, which were empirically developed to determine the effective elastic properties of aligned fiber composites, are used (Halpin 1969). These equations can be expressed as

$$
\begin{gathered}
\frac{\phi_{\mathrm{c}}}{\phi_{\mathrm{m}}}=\frac{1+\xi \eta V_{\mathrm{f}}}{1-\eta V_{\mathrm{f}}}, \\
\eta=\frac{\phi_{\mathrm{f}} / \phi_{m}-1}{\phi_{\mathrm{f}} / \phi_{m}+\xi},
\end{gathered}
$$

274 for which the aspect ratio is $\xi=2 \times\left(l_{\mathrm{f}} / d_{\mathrm{f}}\right)$ with $l_{\mathrm{f}}$ being the fiber length and $d_{\mathrm{f}}$ being the fiber

275 diameter. In Equations (14) and (15), $\phi_{\mathrm{c}}, \phi_{\mathrm{m}}, \phi_{\mathrm{f}}$ refer to composite, matrix and fiber elastic

276 material properties, and $V_{\mathrm{f}}$ is the volume fraction. Following the investigations in the literature, $V_{\mathrm{f}}$ 277 is taken to be within the range from $16 \%$ to $33 \%$ while AS4 carbon fibre is used as the fiber 278 reinforcement with $E_{\mathrm{f} 11}=225000 \mathrm{MPa}, E_{\mathrm{f} 22}=15000 \mathrm{MPa}, G_{\mathrm{f} 12}=15000 \mathrm{MPa}, G_{\mathrm{f} 12}=7000 \mathrm{MPa}$, $279 v_{\mathrm{f} 12}=0.2$, and the matrix material is $3501-6$ epoxy matrix material with $E_{\mathrm{m}}=4200 \mathrm{MPa}, G_{\mathrm{m}}=1567$ $280 \mathrm{MPa}$ and $v_{\mathrm{m}}=0.35$ (Soden et al. 1998; Babu et al. 2018). The RVEs are rectangular prisms $(7 \times 7 \times 4$ 281 ) and the fiber aspect ratio $\xi=3.5$ where both matrix and fiber domains are discretized with C3D4 282 general-purpose tetrahedral elements.

\section{Effective elastic properties of fiber reinforced composites}

286 In this case study, unidirectionally aligned fiber reinforced composite RVEs are generated with 287 RSA code similar to the previous case study. These RVEs are then investigated and their effective 
elastic properties are computed. The fibers and matrix are assumed to be strongly bonded and only

289 the effects of fiber volume fraction on the effective elastic properties are examined. The domain is 290 discretized so that arbitrary meshes are generated on the RVE boundaries $\partial \omega$, as seen in Figure 291 10. Thereafter, six different deformation modes - three uniaxial and shear deformation modes292 are simulated to obtain all the effective elastic parameters that are listed in Table 3. The results 293 show that there is a reinforcing effect of fibers on the elastic properties with a steep increase of $294125 \%$ for $E_{\mathrm{c} 11}, 27 \%$ for $E_{\mathrm{c} 22}$, and $29 \%$ for $E_{\mathrm{c} 33} ; 38 \%$ for $G_{\mathrm{c} 12}, 45 \%$ for $G_{\mathrm{c} 13}$, and $26 \%$ for $G_{\mathrm{c} 23}$; and 295 a decrease of $6 \%$ for $v_{\mathrm{c} 12}, 10 \%$ for $v_{\mathrm{c} 13}$, and $3 \%$ for $v_{\mathrm{c} 23}$ within the investigated volume fraction range. The decrease in $v$ is also experienced with increasing $V_{\mathrm{f}}$ in this case study due to $v_{\mathrm{f}}<v_{\mathrm{m}}$.

297 The percent errors for Young's moduli, shear moduli, and Poisson's ratios between the proposed 298 and analytical solutions are $4.4 \%-13 \%, 0.5 \%-13.2 \%, 0.5 \%-5.1 \%$, respectively.

\section{CONCLUSIONS}

301 In the present study, a data-driven computational homogenization method is presented, the objective of which is to characterize effective material properties directly through their reconstructed microstructures via scanning devices (-e.g., X-ray micro-tomography, etc.). For this purpose, periodic boundary conditions are enforced on the reconstructed microstructures via total distance minimization of control and boundary node sets. Here, the minimization problem, here also referred as Euclidean bipartite matching, is solved with Monte-Carlo simulations which generate random possible solutions and select the solution with the minimum cost. This results in one-to-one matching between the node sets. Thereafter, first order strain driven homogenization is implemented, which, in turn, enables bridging scale-based features and material characterization. In order to understand the performance of the method, first, a convergence study has been conducted on porous RVEs that have non-conformal meshes on their boundaries. It is deduced that

312 the increase in the mesh size after some threshold does not have any significant effect —e.g., the 313 maximum percentile difference of $3.8 \%$ between 185795 (referring to 20 seeds per edge) and 314558311 elements (40 seeds per edge) despite the tremendous increase in time from 423 seconds to 3151992 seconds. Thus, in the present study, 20 seeds per edge is also used in the method verification 316 process. To elaborate, the method is verified by comparing the numerical results computed for 317 realistic renderings of closed cell porous, particle and fiber reinforced composite materials with 318 analytical Hashin-Shtrikman and Halpin-Tsai methods. The percent errors between the analytical 
and computed effective elastic properties are observed to be within acceptable ranges-namely,

$320 \quad 0.04 \%-3.3 \%$ for porous material case, $2.7 \%-17.6 \%$ for particle reinforced composite material case, 321 and $0.5 \%-13.2 \%$ for fiber reinforced composite material case. These results indicate that the 322 proposed data-driven computational homogenization method is a potentially useful tool that can 323 utilize in-situ imaging data at the micro-scale as input, and produce effective properties at the 324 material (meso-) scale.

\section{ACKNOWLEDGMENTS}

The authors gratefully acknowledge the support of Tekniikan edistämissäätiö TES through Foundations' Post Doc Pool, Finland.

Babu, K.P., P.M. Mohite, and C.S. Updahyay. 2018. "Development of an RVE and its stiffness predictions based on mathematical homogenization theory for short fibre composites." International Journal of Solids and Structures 130-131: 80-104. Wilmers. 2018. "Generation of 3D representative volume elements for heterogeneous materials:

338 A review." Progress in Materials Science 96: 322-384.

339 Cerquaglia, M.L., G. Deliége, R. Boman, L. Papeleux, and J.P. Ponthot. 2017. "Reprint of: The 340 particle finite element method for the numerical simulation of bird strike." International Journal of 341 Impact Engineering 72-84.

342 Duval, L., M. Moreaud, C. Couprie, D. Jeulin, H. Talbot, and J. Angulo. 2014. "Image processing 343 for materials characterization: Issues, challenges and opportunities." 2014 IEEE International 344 Conference on Image Processing (ICIP). Paris.

345 Edelsbrunner, H. 1995. "Smooth surfaces for multi-scale shape representation." In Foundations of 346 Software Technology and Theoretical Computer Science, 391-412. Berlin, Heidelberg: Springer.

347 Edelsbrunner, H., D. Kirkpatrick, and R. Seidel. 1983. "On the shape of a set of points in the 348 plane." IEEE Transactions on Information Theory 29 (4): 551 - 559.

349 Evans, J.W. 1993. "Random and cooperative sequential adsorption." Reviews of Modern Physics $35065(1281)$. 
Geers, M.G.D., V.G. Kouznetsova, and W.A.M. Brekelmans. 2010. "Multi-scale computational 352 homogenization: Trends and challenges." Journal of Computational and Applied Mathematics $353 \quad 2175-2182$.

354 Halpin, J.C. 1969. Effects of Environmental Factors on Composite. Ohio: AFML-TR-67-423.

355 Hashin, Z., and S. Shtrikman. 1963. "A Variational Approach to the Elastic Behavior of 356 Multiphase Materials." Journal of the Mechanics and Physics of Solids 11 (2): 127-140.

357 Hernández, J.A., J. Oliver, A.E. Huespe, M.A. Caicedo, and J.C. Cante. 2014. "High-performance 358 model reduction techniques in computational multiscale homogenization." Computer Methods in 359 Applied Mechanics and Engineering 276: 149-189.

360 Hibbitt, Karlsson, and Sorensen. 1992. ABAQUS: Theory Manual. Providence, R.I.: Hibbitt, 361 Karlsson \& Sorensen.

362 Hollister, S.J., and N. Kikuchi. 1994. "Homogenization Theory and Digital Imaging: A Basis for 363 Studying the Mechanics and Design Principles of Bone Tissue." Biotechnology and 364 Bioengineering, Vol. 43: 586-596.

365 Huang, X., and B. Wei. 2010. "Mineral Particle Image Processing and Parameter Extracting." 366 International Conference on Logistics Engineering and Intelligent Transportation System. Wuhan.

367 Hung, M.S., and W.O. Rom. 1980. "Solving the Assignment Problem by Relaxation." Operations 368 Research 28 (4): 969-982.

369 Karakoc, A. 2018. "Sensitivity analysis on the effective stiffness properties of 3-D orthotropic 370 honeycomb cores." International Journal for Computational Methods in Engineering Science and 371 Mechanics 19 (1): 22-30.

372 Karakoc, A, and E Taciroglu. 2017. "Optimal automated path planning for infinitesimal and real373 sized particle assemblies." AIMS Materials Science 4 (4): 847-855.

374 Karakoc, A., E. Hiltunen, and J. Paltakari. 2017. "Geometrical and spatial effects on fiber network 375 connectivity." Composite Structures 168: 335-344.

376 Karakoç, A., P. Tukiainen, J. Freund, and M. Hughes. 2013. "Experiments on the effective 377 compliance in the radial-tangential plane of Norway spruce." Composite Structures 102: 287-293.

378 Kushnevsky, V., O. Morachkovsky, and H. Altenbach. 1998. "Identification of effective properties 379 of particle reinforced composite materials." Computational Mechanics 317-325.

380 Larsson, F., K. Runesson, S. Saroukhani, and R. Vafadari. 2011. "Computational homogenization 381 based on a weak format of micro-periodicity for RVE-problems." Comput. Methods Appl. Mech. 382 Eng. 200: 11-26.

383 Legrain, G., P. Cartraud, I. Perreard, and N. Moes. 2011. "An X- FEM and level set computational 384 approach for image- based modelling: Application to homogenization." International Journal for 385 Numerical Methods in Engineering 86 (7): 915-934. 
Lian, W.D., G. Legrain, and P. Cartraud. 2013. "Image-based computational homogenization and localization: comparison between X-FEM/levelset and voxel-based approaches." Computational Mechanics 51 (3): 279-293.

389 Lopez, E., E. Abisset-Chavanne, C. Ghnatios, S. Comas-Cardona, C. Binetruy, and F. Chinesta. 390 2014. "Towards image-based homogenization by combining scanning techniques and reduced

392 Nazar, A.M., F.A. Silva, and J.J. Ammann. 1996. "Image processing for particle characterization." 393 Materials Characterization 36 (4-5): 165-173.

394 Nguyen, V.D., and L. Noels. 2014. "Computational homogenization of cellular materials." 395 International Journal of Solids and Structures 51 (11-12): 2183-2203.

396 Nguyen, V.D., E. Béchet, C. Geuzaine, and L. Noels. 2012. "Imposing periodic boundary 397 condition on arbitrary meshes by polynomial interpolation." Computational Materials Science 55: 398 390-406.

399 Ren, W., Z. Yang, R. Sharma, C. Zhang, and P.J. Withers. 2015. "Two-dimensional X-ray CT 400 image based meso-scale fracture modelling of concrete." Engineering Fracture Mechanics 133: $401 \quad 24-39$.

402 Rendl, F. 1988. "On the Euclidean assignment problem." Journal of Computational and Applied 403 Mathematics 23 (3): 257-265.

404 Segurado, J., and J. Llorca. 2002. "A numerical approximation to the elastic properties of spherereinforced composites." Journal of the Mechanics and Physics of Solids 2107-2121.

Sjolund, J., A. Karakoc, and J. Freund. 2014. "Accuracy of regular wood cell structure model." Mechanics of Materials 76: 35-44.

Soden, P.D., M.J. Hinton, and A.S. Kaddour. 1998. "Lamina properties, lay-up configurations and loading conditions for a range of fibre-reinforced composite laminates." Compos. Sci. Technol. 58 (7): 1011-1022.

Takano, N., M. Zako, F. Kubo, and K. Kimura. 2003. "Microstructure-based stress analysis and evaluation for porous ceramics by homogenization method with digital image-based modeling."

414 Terada, K., T. Miura, and N. Kikuchi. 1997. "Digital image-based modeling applied to the 415 homogenization analysis of composite materials." Computational Mechanics 20: 331-346.

416 Tyrus, J.M., M. Gosz, and E. DeSantiago. 2007. "A local finite element implementation for 417 imposing periodic boundary conditions on composite micromechanical models." Int. J. Solids 418 Struct. 44 (9): 2972-2989.

419 Yuan, Z., and J. Fish. 2008. "Toward realization of computational homogenization in practice." 420 Int. J. Numer. Methods Eng. 73 (3): 361-380. 
422 Figure 1. Various engineering materials at their material scales and the illustrations of their

423 reconstructed microstructures as representative volume elements (RVEs): (a) closed cell porous

424 material, (b) particle reinforced composite, (c) fiber reinforced composite, (d) nonwoven material.

425

426 Figure 2. Flow chart for the present algorithm and schematic illustration of the boundary and

427 control node matching on a representative volume element RVE based on the proposed method.

428 The term $\partial \omega$ represents the boundaries of the RVE and $\partial \Gamma$ represents the boundaries of

429 rectangular prism bounding the RVE. It is noteworthy that boundaries comprise of vertices, edges

430 and surfaces.

431

432 Figure 3. Illustration of the rectangular prism bounding the RVE and control node sets $(q)$ on its 433 boundaries $(\partial \Gamma)$. The symbols on the right-hand sides of the nodes show the matching sets for the 434 periodicity.

435

$436 \quad$ Figure 4: Details of the control node reduction. $R$ refers to circumradius of the element.

$438 \quad$ Figure 5. Strain driven homogenization with imposed macroscopic strain $\mathbf{e}^{\mathrm{M}}$ and computed stress $439 \mathbf{s}^{\mathrm{M}}$. Here, $\Omega$ and $\partial \Omega$ represent the volume and boundary of continuum, and $\Gamma$ and $\partial \Gamma$ represent 440 the volume and boundary of the rectangular prism that bounds the RVE.

442 Figure 6. Mesh size investigations: (a) coarsest mesh (I), optimum mesh comprising the CPU time 443 and properties (II), finest mesh (III), (b) effect of mesh size on the convergence of effective elastic 444 properties and CPU time. 
446 Figure 7. Control node investigations: (a) representative volume element RVE with 152 control 447 nodes (I), 2402 nodes (II), 3628 nodes (III), (b) effect of number of control nodes on the effective 448 elastic properties and CPU time.

449

$450 \quad$ Figure 8. Deformation modes of the closed cell porous material: (a) top and bottom isometric 451 views, (b) uniaxial deformation modes, (c) shear deformation modes. A deformation scale factor 452 of 10 was used for better illustration.

454 Figure 9. Deformation modes of spherical particle reinforced composite: (a) isometric view, (b) 455 generated arbitrary mesh on the RVE boundaries, (c) uniaxial deformation modes, (d) shear 456 deformation modes. A deformation scale factor of 10 was used for better illustration.

458 Figure 10. Deformation modes of short fiber reinforced composite: (a) isometric view, (b) 459 generated arbitrary mesh on the RVE boundaries, (c) uniaxial deformation modes, (d) shear 460 deformation modes. A deformation scale factor of 5 was used for better illustration. 
473 Table 1. Effect of void volume fraction $V_{f}$ on the effective elastic properties of closed cell porous 474 materials. HS refers to Hashin-Shtrikman. Perc. Diff. refers to the percentile difference between 475 the mean values of the simulations and ones from Hashin-Shtrikman equations.

\begin{tabular}{|c|c|c|c|c|c|c|c|c|c|c|}
\hline $\begin{array}{r}V_{\mathrm{f}} \\
(\%) \\
\end{array}$ & & $E_{\mathrm{c} 11}$ & $E_{\mathrm{c} 22}$ & $\begin{array}{c}E_{\mathrm{c} 33} \\
(\mathrm{MPa})\end{array}$ & $\begin{array}{r}G_{\mathrm{c} 12} \\
(\mathrm{MPa}) \\
\end{array}$ & $\begin{array}{c}G_{\mathrm{c} 13} \\
(\mathrm{MPa})\end{array}$ & $\begin{array}{c}G_{\mathrm{c} 23} \\
(\mathrm{MPa})\end{array}$ & $v_{\mathrm{c} 12}$ & $v_{\mathrm{c} 13}$ & $v_{\mathrm{c} 23}$ \\
\hline \multirow[t]{7}{*}{10} & RVE 1 & 2456.4 & 2420.6 & 2444.8 & 900.9 & 904.5 & 903.3 & 0.35 & 0.35 & 0.3 \\
\hline & & 2463.3 & 2430.7 & 2477.7 & 904.1 & 903.9 & 903.3 & & 35 & 0.35 \\
\hline & E 3 & 497.5 & 2397.0 & 2451.5 & 900.3 & 906.9 & 96.6 & & 36 & 0.35 \\
\hline & Mean & 2472.4 & 2416.1 & 2458.0 & 901.8 & 905.1 & 901.1 & & 35 & 0.35 \\
\hline & Dev & 22.0 & 17.3 & 17.4 & 2.0 & 1.6 & 3.9 & 0.02 & 0.02 & 0.01 \\
\hline & & 2457.6 & 2457.6 & 2457.6 & 903.9 & 903.9 & 903.9 & 0.36 & 0.36 & 0.36 \\
\hline & & 0.6 & 1.7 & 0.04 & 0.2 & 0.1 & 0.3 & 1.4 & 1.7 & 3.3 \\
\hline \multirow[t]{7}{*}{15} & $\mathrm{RV}$ & 2242.5 & 2243.5 & 2251.7 & 823.6 & 829.7 & 828.5 & 0.34 & 0.35 & 0.34 \\
\hline & & 279.0 & 2280.2 & 2243.3 & 844.1 & & & 0.35 & 35 & 0.35 \\
\hline & & 2281.6 & 2267.7 & 2296.5 & 847.1 & & & 0.35 & 35 & 0.34 \\
\hline & & 2267.7 & 2263 & 2263 & 838 & 0.3 & & & 35 & 0.34 \\
\hline & & 21.9 & 18 & 28 & & & & & 00 & 0.00 \\
\hline & & 2212.4 & 2212.4 & 2212.4 & 819.4 & 819 & 819.4 & & 0.35 & 0.35 \\
\hline & & 2.4 & 2.3 & 2.3 & 2. & 2. & 2.0 & & 0.7 & 1.9 \\
\hline \multirow[t]{7}{*}{20} & & 2062.3 & 2029.5 & 2052.0 & 759.0 & 763.2 & 759.7 & 34 & 0.34 & 0.34 \\
\hline & & 59.8 & 0.8 & 204 & 753 & & & 4 & 34 & 0.34 \\
\hline & & 89.6 & 1930.0 & 1980.4 & 738.0 & 744.3 & 737.0 & 4 & 34 & 0.33 \\
\hline & & 2037.2 & 1990.1 & 2027.2 & 750.3 & 752.1 & 749.3 & 0.34 & 34 & 0.33 \\
\hline & & 41.3 & 52.9 & 40.5 & 10.9 & & 11.5 & & 00 & 0.00 \\
\hline & & 2004.7 & 2004.7 & 2004.7 & 746.8 & 746.8 & 746.8 & 0. & 0.34 & 0.34 \\
\hline & & 1.6 & 0.7 & 1.1 & 5 & 7 & 0.3 & & 1.0 & 2.3 \\
\hline \multirow[t]{7}{*}{25} & & & & & & & & & 33 & 0.33 \\
\hline & & 03.9 & 1841.6 & 1869.5 & 709.3 & 707 & 700.6 & 0.3 & 0.34 & 0.33 \\
\hline & & 1800.8 & 1795.5 & 1763.3 & 673.2 & 665.3 & 664.4 & 0.33 & 0.33 & 0.33 \\
\hline & & 1858.7 & 1828.6 & 1817.1 & 687.2 & 685.2 & 683.5 & 0.3 & 0.33 & 0.33 \\
\hline & & 52.7 & & & 19.4 & 21.0 & 18.2 & 1 & 00 & 0.00 \\
\hline & $\mathrm{H}$ & 1805.1 & 1805.1 & 1805.1 & 676.3 & 676.3 & 676.3 & 0.33 & 0.33 & 0.33 \\
\hline & Perc. Diff. (\%) & 2.9 & 1.3 & 0.7 & 1.6 & 1.3 & 1.1 & 0.8 & 0.6 & 1.4 \\
\hline \multirow[t]{7}{*}{28} & & 1793.9 & 1703.2 & & & & 650.5 & 0.34 & 0.33 & 0.32 \\
\hline & & & & 170 & & & & & 0.33 & 0.33 \\
\hline & & & & & & & & & 33 & 0.32 \\
\hline & & 1774.9 & 1737.1 & 1740.5 & 660 & & 653.5 & 0.3 & 33 & 0.32 \\
\hline & & & & & & & & 0.0 & .00 & 0.00 \\
\hline & & 1729.6 & & 172 & & & 649.4 & 0. & 0.33 & 0.33 \\
\hline & Perc. Diff. (\%) & 2.5 & 0.4 & 0.6 & 1.6 & 1.3 & 0.6 & 0.2 & 0.2 & 2.1 \\
\hline
\end{tabular}


478 Table 2. Effect of particle volume fraction on the effective elastic properties of spherical particle 479 reinforced composites. HS refers to Hashin-Shtrikman. Perc. Diff. refers to the percentile 480 difference between the mean values of the simulations and ones from Hashin-Shtrikman equations. 481

\begin{tabular}{|c|c|c|c|c|c|c|c|c|c|c|}
\hline$(\%)$ & & $\begin{array}{c}E_{\mathrm{c} 11} \\
(\mathrm{MPa})\end{array}$ & $\begin{array}{c}E_{\mathrm{c} 22} \\
(\mathrm{MPa})\end{array}$ & $\begin{array}{c}E_{\mathrm{c} 33} \\
(\mathrm{MPa})\end{array}$ & $\begin{array}{c}G_{\mathrm{c} 12} \\
(\mathrm{MPa})\end{array}$ & $\begin{array}{c}G_{\mathrm{cl3}} \\
(\mathrm{MPa})\end{array}$ & $\begin{array}{c}G_{\mathrm{c} 23} \\
(\mathrm{MPa})\end{array}$ & $v_{\mathrm{c} 12}$ & $v_{\mathrm{c} 13}$ & $v_{\mathrm{c} 23}$ \\
\hline \multirow[t]{7}{*}{10} & RVE 1 & 3753.2 & 3733.3 & 3729.3 & 1366.4 & 1361.9 & 1357.4 & 0.36 & 0.37 & 0.37 \\
\hline & RVE 2 & 3809.2 & 3750.5 & 3772.8 & 1371.7 & 1377.3 & 1369.6 & 0.37 & 0.36 & 0.37 \\
\hline & RVE 3 & 3775.3 & 3798.8 & 3792.7 & 1384.0 & 1392.8 & 1378.2 & 0.36 & 0.37 & 0.36 \\
\hline & Mean & 3779.2 & 3760.8 & 3764.9 & 1374.0 & 1377.3 & 1368.4 & 0.36 & 0.37 & 0.37 \\
\hline & Std Dev & 28.2 & 34.0 & 32.4 & 9.0 & 15.4 & 10.5 & 0.00 & 0.00 & 0.00 \\
\hline & HS-Lower & 3648.6 & 3648.6 & 3648.6 & 1331.1 & 1331.1 & 1331.1 & 0.37 & 0.37 & 0.37 \\
\hline & Perc. Diff. (\%) & 3.5 & 3.0 & 3.1 & 3.1 & 3.4 & 2.7 & 1.6 & 1.2 & 1.5 \\
\hline \multirow[t]{7}{*}{15} & RVE 1 & 4266.5 & 4242.9 & 4265.3 & 1549.8 & 1543.1 & 1568.5 & 0.36 & 0.36 & 0.36 \\
\hline & RVE 2 & 4326.9 & 4265.3 & 4306.5 & 1540.0 & 1557.4 & 1535.5 & 0.36 & 0.36 & 0.36 \\
\hline & RVE 3 & 4168.1 & 4148.4 & 4149.6 & 1497.1 & 1512.1 & 1506.9 & 0.35 & 0.36 & 0.36 \\
\hline & Mean & 4253.8 & 4218.8 & 4240.5 & 1529.0 & 1537.5 & 1537.0 & 0.36 & 0.36 & 0.36 \\
\hline & Std Dev & 80.1 & 62.0 & 81.4 & 28.0 & 23.2 & 30.8 & 0.00 & 0.00 & 0.00 \\
\hline & HS-Lower & 4023.3 & 4023.3 & 4023.3 & 1472.8 & 1472.8 & 1472.8 & 0.37 & 0.37 & 0.37 \\
\hline & Perc. Diff. (\%) & 5.4 & 4.6 & 5.1 & 3.7 & 4.2 & 4.2 & 2.7 & 2.3 & 2.0 \\
\hline \multirow[t]{7}{*}{20} & RVE 1 & 4853.3 & 4873.5 & 4931.8 & 1732.7 & 1717.8 & 1776.3 & 0.35 & 0.34 & 0.35 \\
\hline & RVE 2 & 4920.3 & 4944.5 & 4940.9 & 1760.5 & 1794.8 & 1806.8 & 0.34 & 0.35 & 0.35 \\
\hline & RVE 3 & 5003.4 & 4983.4 & 5055.6 & 1781.8 & 1829.1 & 1825.7 & 0.34 & 0.34 & 0.35 \\
\hline & Mean & 4925.7 & 4933.8 & 4976.1 & 1758.4 & 1780.5 & 1802.9 & 0.34 & 0.35 & 0.35 \\
\hline & Std Dev & 75.2 & 55.7 & 69.0 & 24.6 & 57.0 & 24.9 & 0.00 & 0.01 & 0.00 \\
\hline & HS-Lower & 4439.1 & 4439.1 & 4439.1 & 1630.4 & 1630.4 & 1630.4 & 0.36 & 0.36 & 0.36 \\
\hline & Perc. Diff. (\%) & 9.9 & 10.0 & 10.8 & 7.3 & 8.4 & 9.6 & 5.3 & 4.6 & 4.0 \\
\hline \multirow[t]{7}{*}{25} & RVE 1 & 5630.5 & 5705.4 & 6191.6 & 2057.0 & 2100.9 & 2130.1 & 0.35 & 0.31 & 0.31 \\
\hline & RVE 2 & 5683.1 & 5820.2 & 5631.5 & 2087.1 & 2019.4 & 2055.3 & 0.33 & 0.34 & 0.34 \\
\hline & RVE 3 & 5776.8 & 5966.4 & 5851.2 & 2117.7 & 2116.1 & 2216.0 & 0.32 & 0.34 & 0.34 \\
\hline & Mean & 5696.8 & 5830.6 & 5891.5 & 2087.2 & 2078.8 & 2133.8 & 0.33 & 0.33 & 0.33 \\
\hline & Std Dev & 74.1 & 130.8 & 282.2 & 30.4 & 52.0 & 80.4 & 0.01 & 0.02 & 0.02 \\
\hline & HS-Lower & 4903.3 & 4903.3 & 4903.3 & 1806.9 & 1806.9 & 1806.9 & 0.36 & 0.36 & 0.36 \\
\hline & Perc. Diff. (\%) & 13.9 & 15.9 & 16.8 & 13.4 & 13.1 & 15.3 & 7.1 & 8.1 & 8.8 \\
\hline \multirow[t]{7}{*}{28} & RVE 1 & 6201.6 & 5985.4 & 6131.5 & 2148.5 & 2247.3 & 2129.8 & 0.33 & 0.34 & 0.33 \\
\hline & RVE 2 & 6567.9 & 6643.9 & 6631.7 & 2391.3 & 2390.8 & 2438.7 & 0.32 & 0.33 & 0.32 \\
\hline & RVE 3 & 6341.2 & 6167.5 & 6338.8 & 2252.2 & 2218.1 & 2209.2 & 0.34 & 0.32 & 0.32 \\
\hline & Mean & 6370.2 & 6265.6 & 6367.3 & 2264.0 & 2285.4 & 2259.3 & 0.33 & 0.33 & 0.32 \\
\hline & Std Dev & 184.9 & 340.1 & 251.3 & 121.8 & 92.4 & 160.4 & 0.01 & 0.01 & 0.00 \\
\hline & HS-Lower & 5251.3 & 5251.3 & 5251.3 & 1939.6 & 1939.6 & 1939.6 & 0.35 & 0.35 & 0.35 \\
\hline & Perc. Diff. (\%) & 17.6 & 16.2 & 17.5 & 14.3 & 15.1 & 14.2 & 7.1 & 8.0 & 9.1 \\
\hline
\end{tabular}


483 Table 3. Effect of fiber volume fraction on the effective elastic properties of fibre reinforced 484 composites. Perc. Diff. refers to the percentile difference between the values of the simulations 485 and ones from Halpin-Tsai equations.

486

\begin{tabular}{|c|c|c|c|c|c|c|c|c|c|c|}
\hline $\begin{array}{c}V_{\mathrm{f}} \\
(\%)\end{array}$ & & $\begin{array}{c}E_{\mathrm{c} 11} \\
(\mathrm{MPa})\end{array}$ & $\begin{array}{c}E_{\mathrm{c} 22} \\
(\mathrm{MPa})\end{array}$ & $\begin{array}{c}E_{\mathrm{c} 33} \\
(\mathrm{MPa})\end{array}$ & $\begin{array}{c}G_{\mathrm{c} 12} \\
(\mathrm{MPa})\end{array}$ & $\begin{array}{c}G_{\mathrm{c} 13} \\
(\mathrm{MPa})\end{array}$ & $\begin{array}{c}G_{\mathrm{c} 23} \\
(\mathrm{MPa})\end{array}$ & $v_{\mathrm{c} 12}$ & $v_{\mathrm{c} 13}$ & $v_{\mathrm{c} 23}$ \\
\hline \multirow[t]{3}{*}{16} & RVE & 8880.4 & 5052.5 & 5010.7 & 1993.3 & 2089.7 & 1862.9 & 0.32 & 0.31 & 0.38 \\
\hline & Halpin-Tsai & 10205.2 & 5423.9 & 5423.9 & 2025.5 & 2025.5 & 1914.5 & 0.32 & 0.32 & - \\
\hline & Perc. Diff. (\%) & 13.0 & 6.8 & 7.6 & 1.6 & 3.2 & 2.7 & 0.5 & 3.9 & - \\
\hline \multirow[t]{3}{*}{17} & RVE & 10337.9 & 5246.6 & 5156.3 & 2089.9 & 2127.6 & 1874.1 & 0.31 & 0.31 & 0.38 \\
\hline & Halpin & 10872.0 & 5549.4 & 5549.4 & 2074.8 & 2074.8 & 1950.6 & 0.32 & 0.32 & - \\
\hline & Perc. & 4.9 & 5.5 & 7.1 & 0.7 & 2.5 & 3.9 & 1.9 & 2.3 & - \\
\hline \multirow[t]{3}{*}{19} & RVE & 10403.0 & 5292.2 & 5249.6 & 2104.2 & 2221.3 & 1921.5 & 0.31 & 0.31 & 0.38 \\
\hline & Halpir & 11499.3 & 5665.8 & 5665.8 & 2121.0 & 2121.0 & 1984.2 & 0.32 & 0.3 & - \\
\hline & & 9.5 & 6.6 & 7.3 & 0.8 & 4.7 & 3.2 & 0.6 & & - \\
\hline \multirow[t]{3}{*}{20} & RVE & 11415.6 & 5361.2 & 5397.6 & 2151.7 & 2362.8 & 1954.3 & 0.32 & & 0.38 \\
\hline & Halpir & 12142.2 & 5783.3 & 5783.3 & 2168.0 & 2168.0 & 2018.2 & 0.31 & 0.3 & - \\
\hline & & 6.0 & 7.3 & 6.7 & 0.8 & 9.0 & 3.2 & 1.7 & & - \\
\hline \multirow[t]{3}{*}{23} & RVE & 12390.0 & 5585.9 & 5552.8 & 2255.9 & 2465.6 & 2032.9 & 0.31 & 0.3 & 0.38 \\
\hline & Halpir & 13747.8 & 6069.6 & 6069.6 & 2284.3 & 2284.3 & 2101.4 & 0.31 & 0.31 & - \\
\hline & & & & & & & & & & - \\
\hline \multirow[t]{3}{*}{25} & RVE & 13999.0 & 5771.3 & 5782.4 & 2410.3 & 2650.3 & 2100.3 & 0.31 & 0.30 & 0.37 \\
\hline & Halpir & 15049.1 & 6294.2 & 6294.2 & 2377.3 & 2377.3 & 2167.0 & 0.30 & 0.3 & - \\
\hline & Perc. D & 7.0 & 8.3 & 8.1 & 1.4 & 11.5 & 3.1 & 1.5 & & - \\
\hline \multirow[t]{3}{*}{27} & RVE & 14391.6 & 5971.6 & 5927.4 & 2477.8 & 2643.9 & 2184.2 & 0.30 & 0.29 & 0.37 \\
\hline & & 16291.0 & 6502.7 & 6502.7 & 2465.1 & 2465.1 & 2228.1 & 0.30 & 0.30 & - \\
\hline & Perc. Diff. (\%) & 11.7 & 8.2 & 8.8 & 0.5 & 7.3 & 2.0 & 2.0 & 3.1 & - \\
\hline \multirow[t]{3}{*}{29} & RVE & 16757.7 & 6090.6 & 6164.2 & 2569.6 & 2887.7 & 2230.6 & 0.30 & 0.29 & 0.37 \\
\hline & & 17523.1 & 6704.1 & 6704.1 & 2551.2 & 2551.2 & 2287.5 & 0.30 & 0.30 & - \\
\hline & Perc. Diff. (\%) & 4.4 & 9.2 & 8.1 & 0.7 & 13.2 & 2.5 & 1.0 & 2.3 & - \\
\hline \multirow[t]{3}{*}{30} & RVE & 15688.3 & 6169.1 & 6228.0 & 2565.5 & 2885.9 & 2221.2 & 0.30 & 0.29 & 0.36 \\
\hline & & 17851.9 & 6757.0 & 6757.0 & 2574.0 & 2574.0 & 2303.1 & 0.30 & 0.30 & - \\
\hline & & & 8.7 & 7.8 & 0.3 & 12.1 & 3.6 & 0.7 & 3.3 & - \\
\hline \multirow[t]{3}{*}{33} & RVE & 19945.3 & 6418.7 & 6484.8 & 2755.6 & 3023.3 & 2340.0 & 0.30 & 0.28 & 0.37 \\
\hline & & 20171.7 & 7119.7 & 7119.7 & 2733.1 & & 2410.7 & 0.29 & 0.29 & - \\
\hline & Perc. Diff. (\%) & 1.1 & 9.8 & 8.9 & 0.8 & 10.6 & 2.9 & 3.4 & 5.1 & - \\
\hline
\end{tabular}


(a)

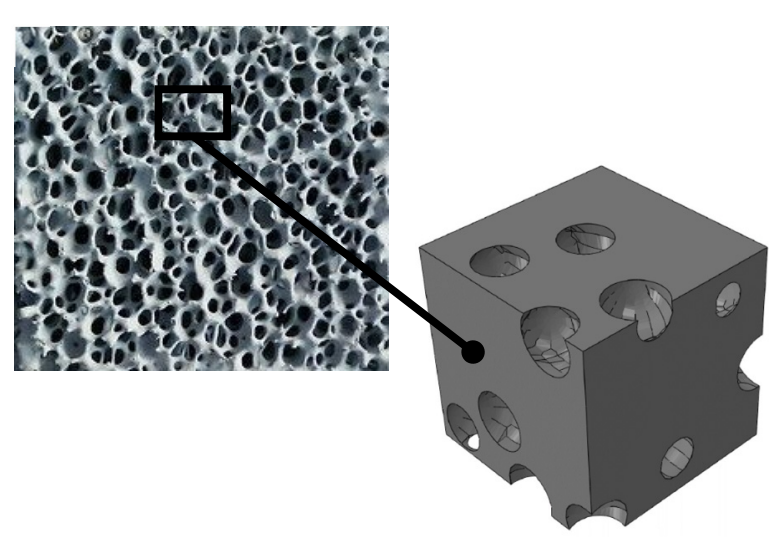

(c)

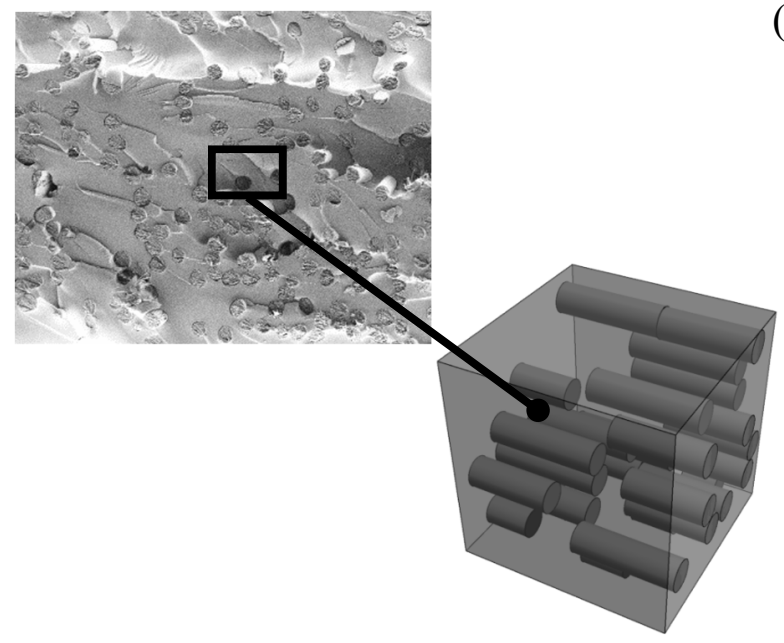

(b)

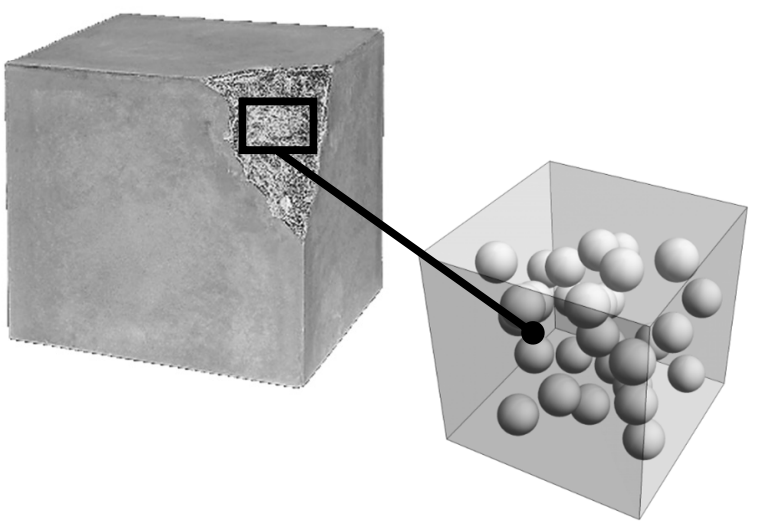

(d)

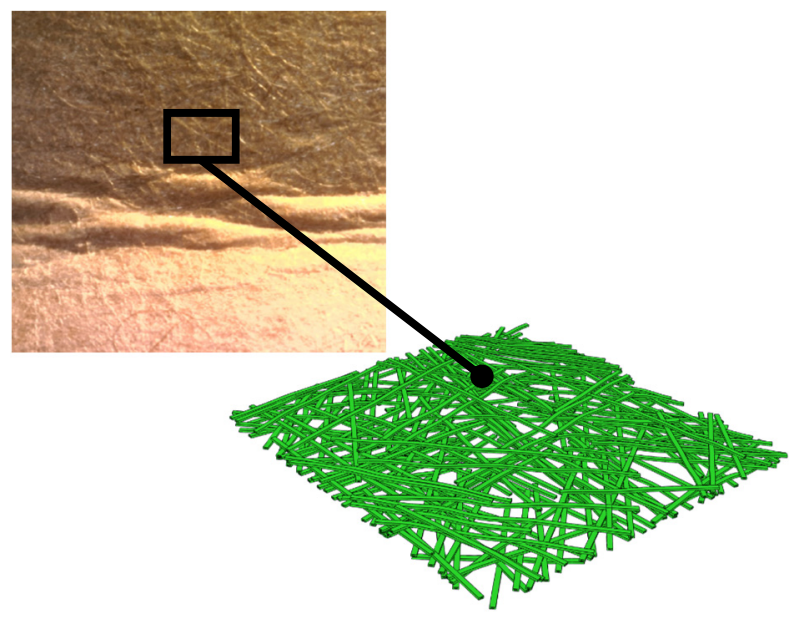



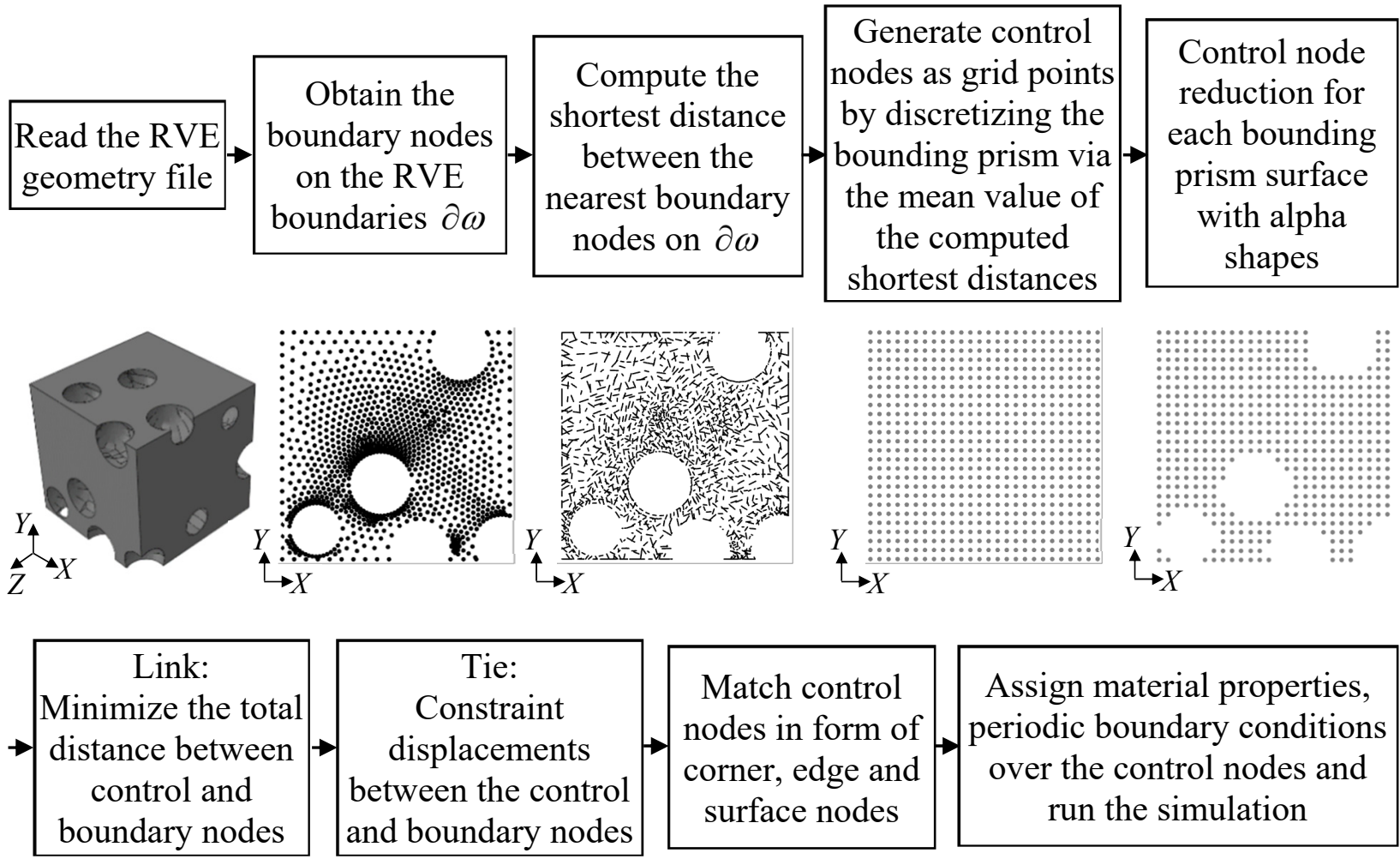

Assign material properties, periodic boundary conditions over the control nodes and run the simulation
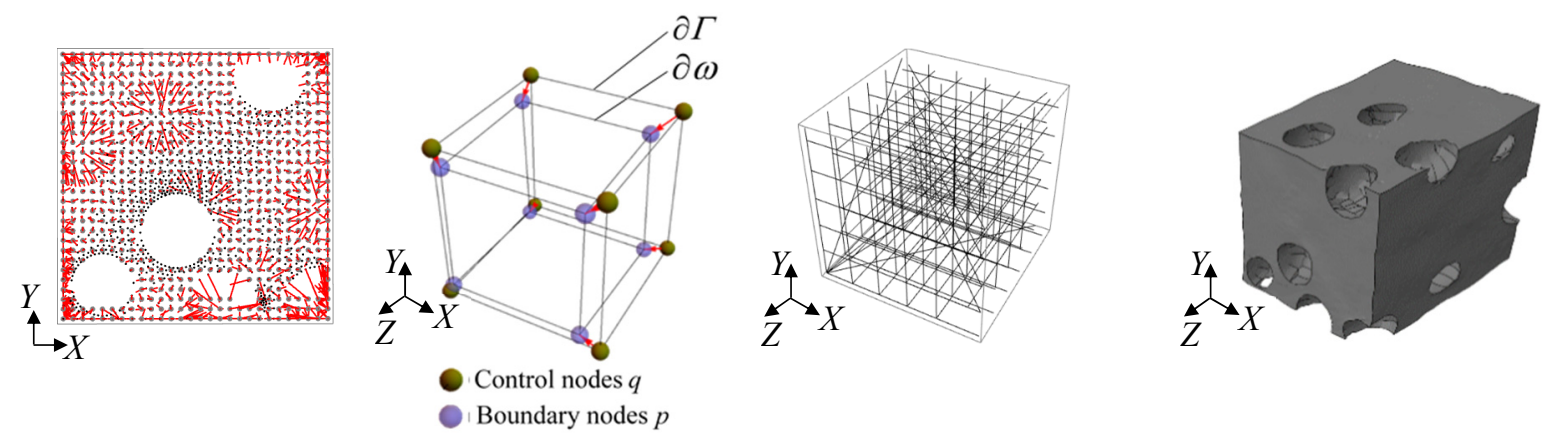


\begin{tabular}{|c|c|c|c|c|c|}
\hline & Corner nodes & Edge nodes & & Surface nodes & \\
\hline $\begin{array}{l}\mathrm{A}_{1}^{\prime \prime} \\
\rightarrow X\end{array}$ & $\begin{array}{l}\text { Node at point } A \\
\text { Node at point } B \\
\text { Node at point } C \\
\text { Node at point } D \\
\text { Node at point } A_{1} \\
\text { Node at point } B_{1} \\
\text { Node at point } C_{1} \\
\text { Node at point } D_{1}\end{array}$ & $\begin{array}{l}\text { Nodes on edge } A B \\
\text { Nodes on edge } B C \\
\text { Nodes on edge } D C \\
\text { Nodes on edge } A D \\
\text { Nodes on edge } A_{1} B_{1} \\
\text { Nodes on edge } B_{1} C_{1} \\
\text { Nodes on edge } D_{1} C_{1} \\
\text { Nodes on edge } A_{1} D_{1} \\
\text { Nodes on edge } A_{1} A \\
\text { Nodes on edge } B_{1} B \\
\text { Nodes on edge } C_{1} C \\
\text { Nodes on edge } D_{1} D\end{array}$ & $\begin{array}{l}\dot{\bullet} \\
\dot{0} \\
\dot{0} \\
\dot{0} \\
\dot{ } \\
0 \\
0 \\
0 \\
0 \\
0\end{array}$ & $\begin{array}{l}\text { Nodes on surface } A B C D \\
\text { Nodes on surface } A_{1} B_{1} C_{1} D_{1} \\
\text { Nodes on surface } A A_{1} D_{1} D \\
\text { Nodes on surface } B B_{1} C_{1} C \\
\text { Nodes on surface } D_{1} C_{1} C \\
\text { Nodes on surface } A A_{1} B_{1} B\end{array}$ & $\begin{array}{l}\dot{\bullet} \\
\dot{\bullet} \\
\mathbf{0} \\
\square \\
\Delta\end{array}$ \\
\hline
\end{tabular}




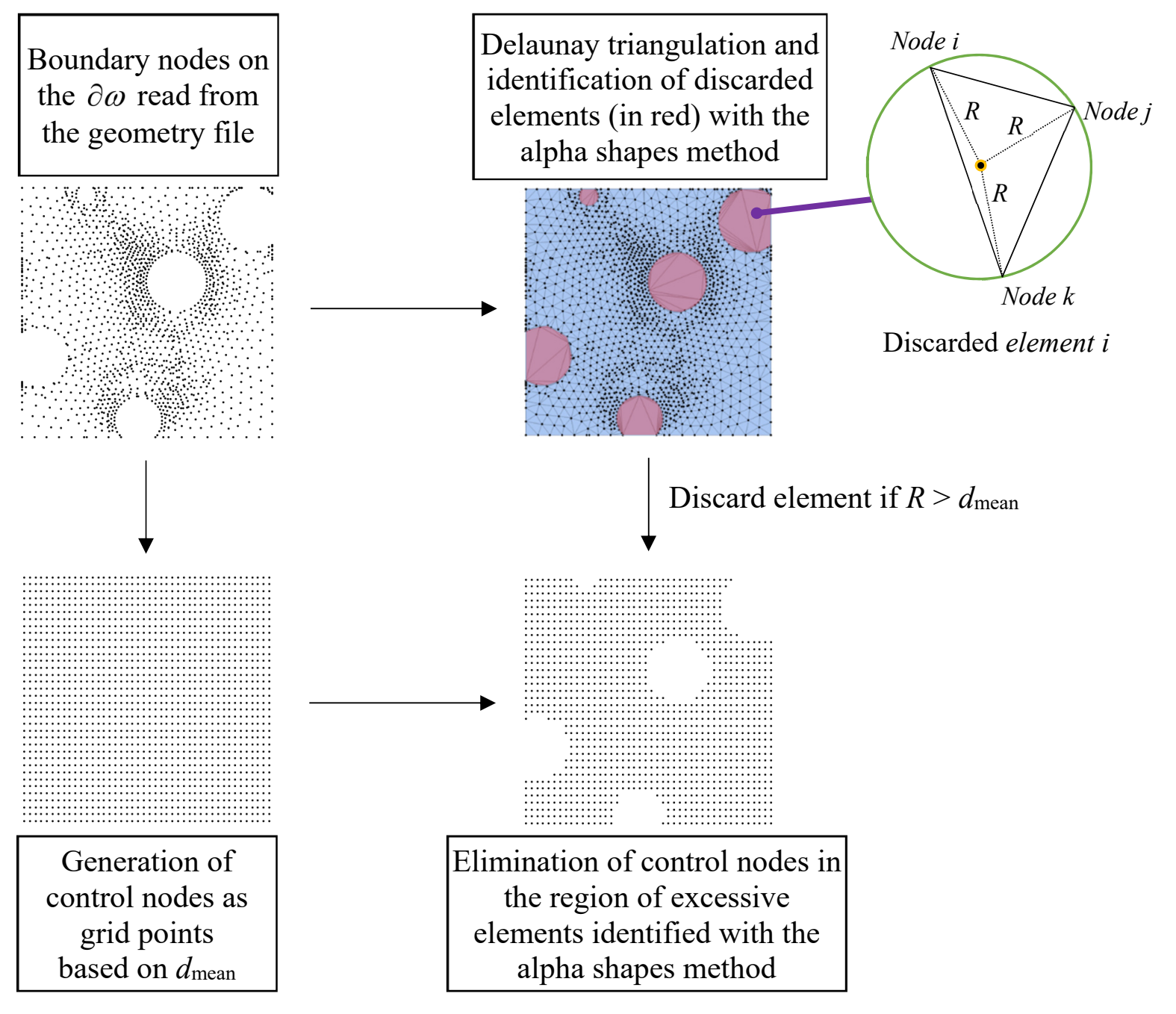




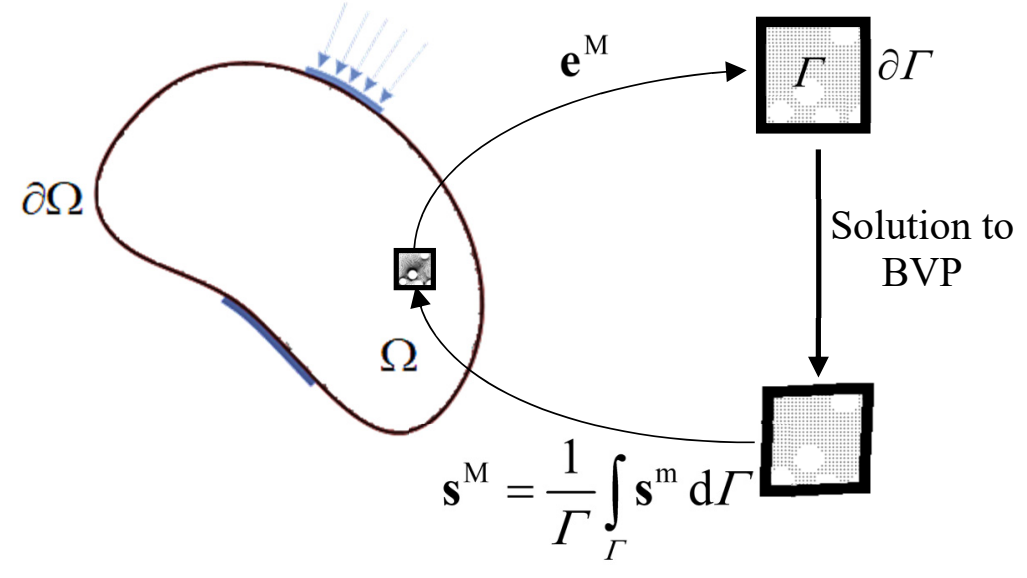


(a)
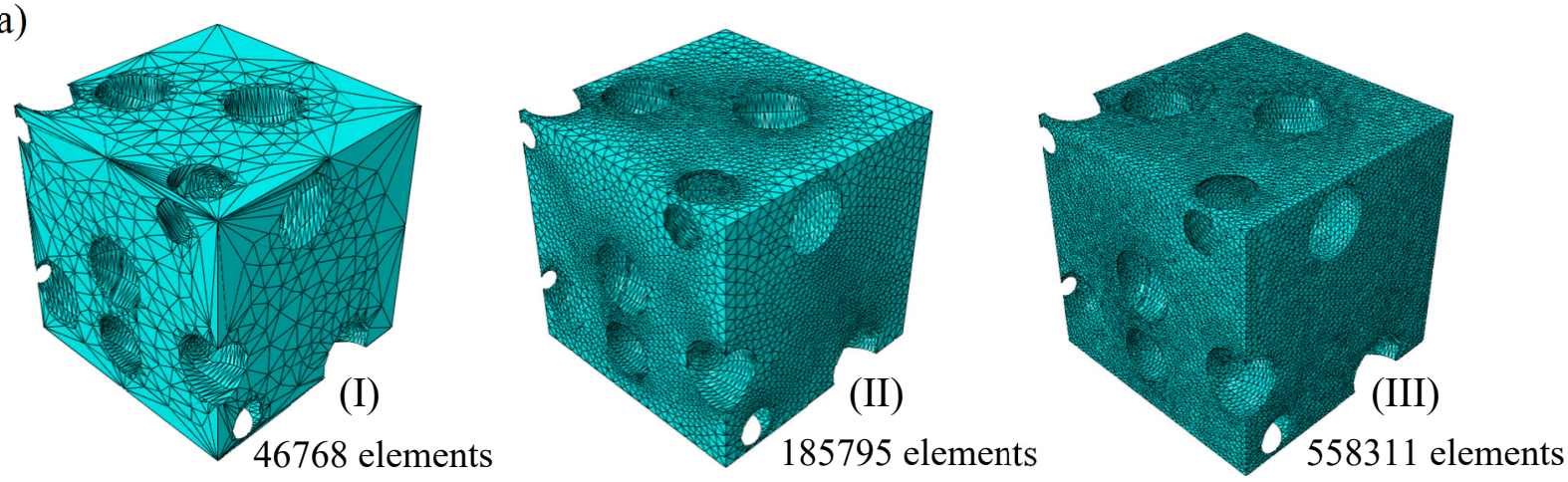

(b)
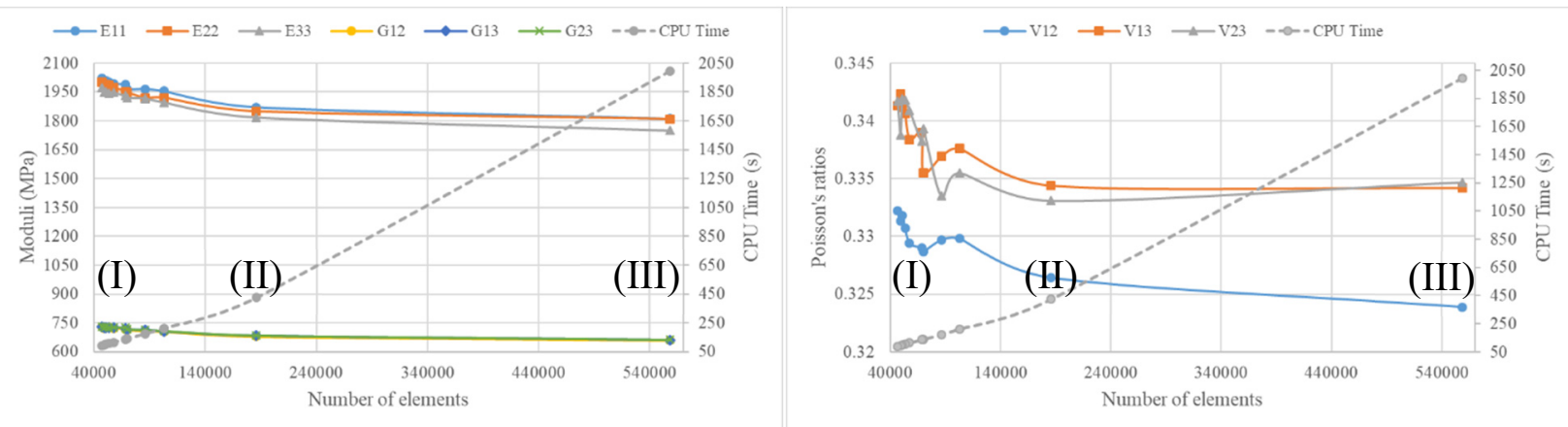


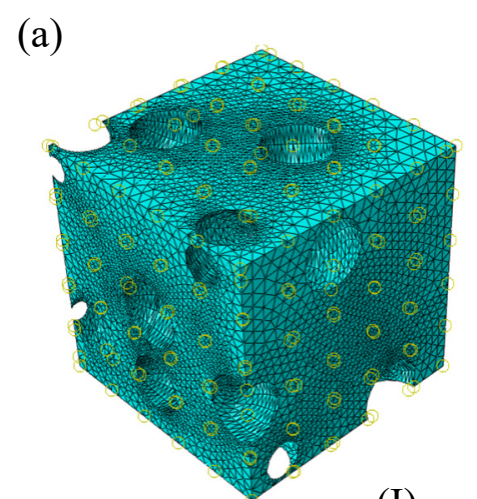

(I)

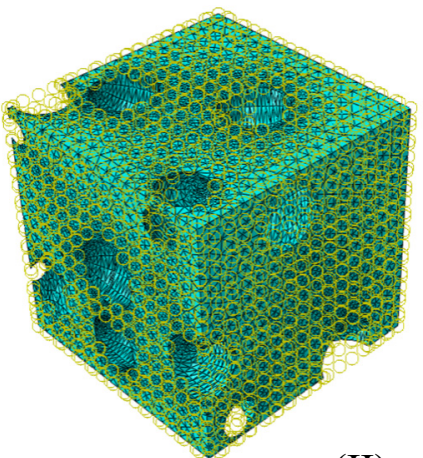

(II)

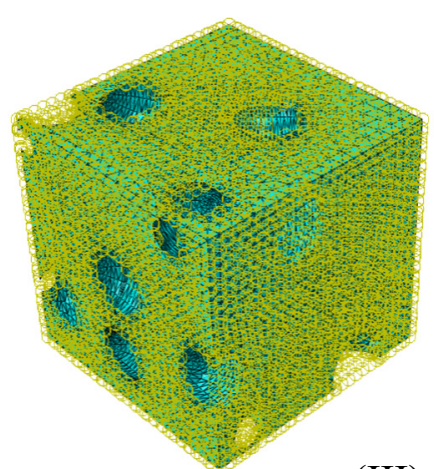

(III)

152 control nodes

2402 control nodes

3628 control nodes

(b)
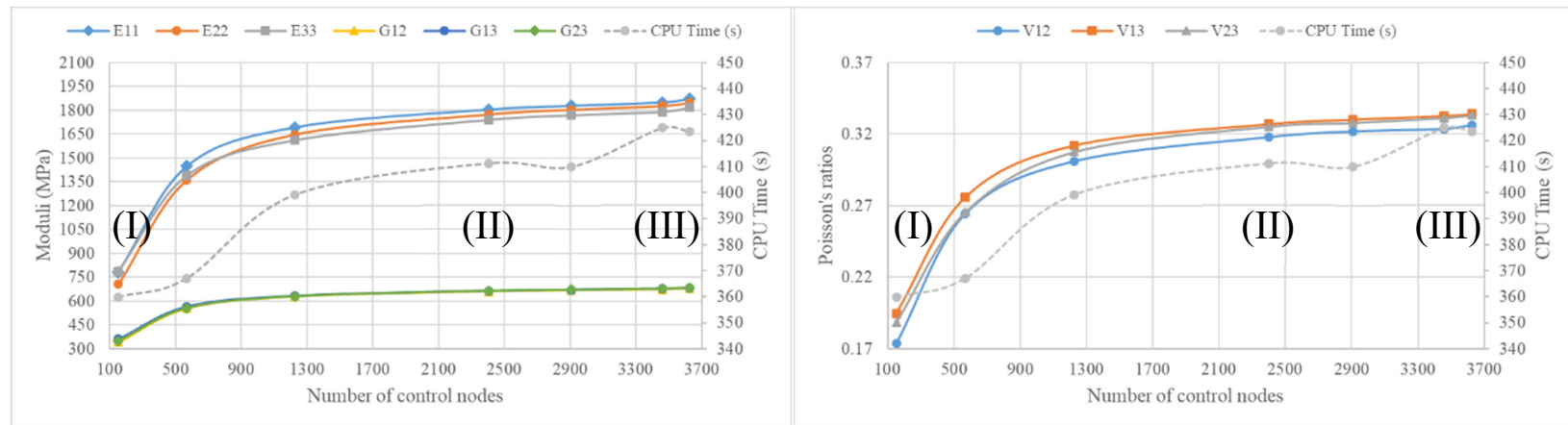


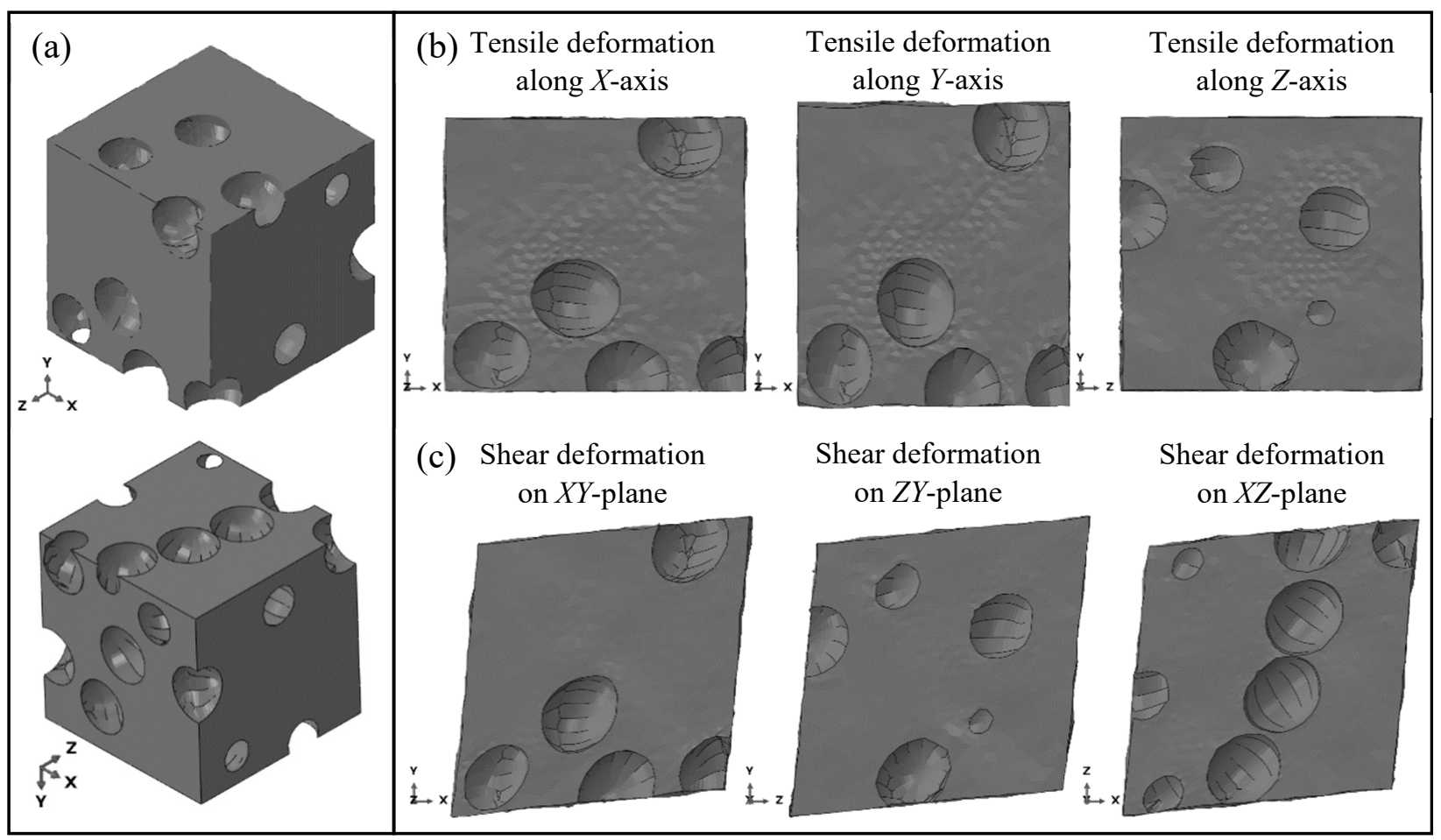




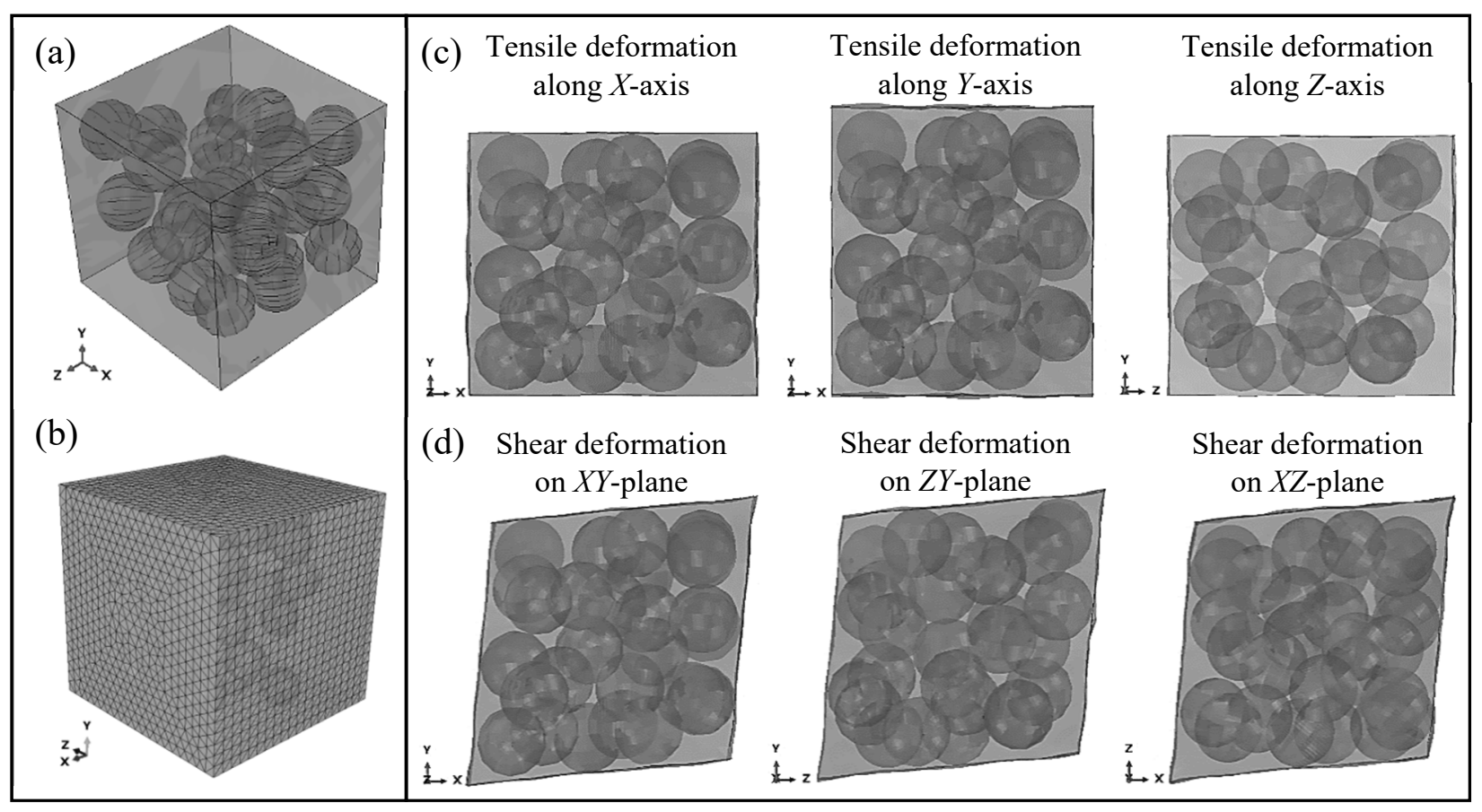




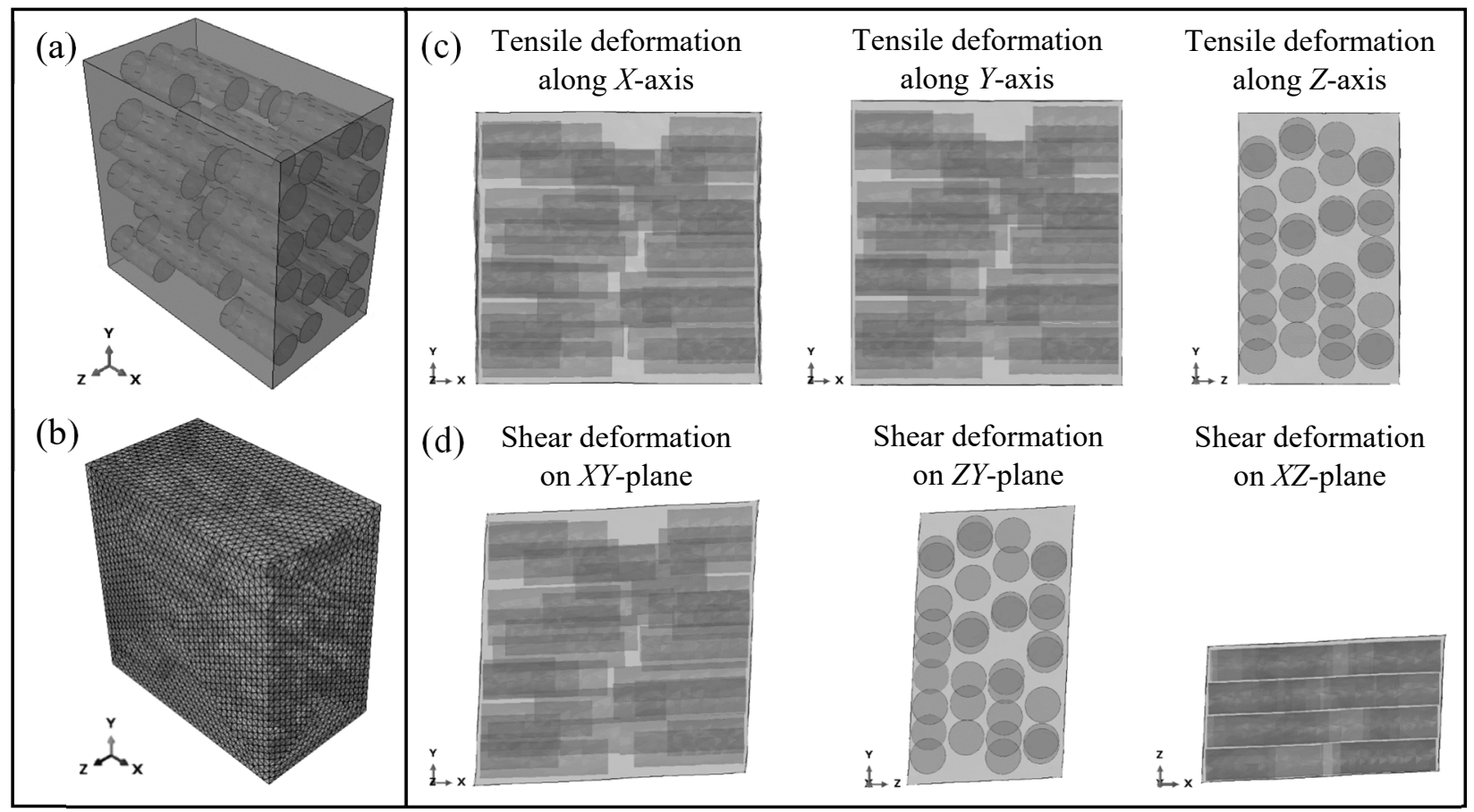

